\title{
Societies Consortium on Sexual Harassment in STEMM: MRS joins academic and professional societies as inaugural member
}

$\mathrm{T}_{\mathrm{h}=\mathrm{s}}^{\mathrm{h}}$ he Materials Research Society (MRS) has joined with more than 80 leading academic and professional societies as an inaugural member of the Societies Consortium on Sexual Harassment in STEMM (science, technology, engineering, mathematics, and medicine) to advance professional and ethical conduct, climate, and culture across their respective fields. The establishment of the Societies Consortium reflects a shared understanding that professional societies have a unique responsibility in their role as standard-setters for STEMM fields to address the pervasive problem of sexual and gender harassment.

The Societies Consortium was established by the American Association for the Advancement of Science (AAAS), the Association of American Medical
Colleges (AAMC), and the American Geophysical Union (AGU), with Education Counsel serving as the policy and law consultant for the initiative. All 80+ participating societies have agreed to help develop and deploy high-impact resources for societies and others in STEMM fields.

"This consortium provides both leadership for a broad diversity of our societies' collective voices and actions to advance ethics, equity, inclusion, and excellence in STEMM research, education, and practice," said Shirley Malcom, senior advisor at AAAS.

Focused on advancing full participation and excellence in STEMM and preventing and responding to sexual and gender harassment in STEMM environments, the Societies Consortium will develop research- and evidence-based resources for societies that include findings and expertise from social and behavioral sciences.

"Since our founding, MRS' core value is to be broadly inclusive and egalitarian," said Todd M. Osman, MRS Executive Director. "MRS has a longstanding tradition of support for inclusion and diversity in the scientific and engineering community. Participation in the Societies Consortium is a next logical step for MRS, collectively working with peer organizations who embrace diversity as a driving force for innovation, excellence, and new discoveries."

For more information on the Societies Consortium on Sexual Harassment in STEMM, contact societiesconsortium@ educationcounsel.com.

Asilomar Bioelectronics Symposium 2019 scheduled for September 3-7 asilomarbioelectronics.org

The Asilomar Bioelectronics September 3-7 in Pacific Grove, Calif. This interactive symposium will focus on defining the frontiers of bioelectronics and mapping future directions. Organizers are Marco Rolandi, University of California, Santa Cruz; Aleksandr Noy, Lawrence Livermore National Laboratory; Jonathan Rivnay,
Northwestern University; and Sahika Inal, King Abdullah University of Science and Technology.

Modern electronics and materials science are bringing revolutionary advances to biointerface design and are shattering the limits of what is possible in the areas of biomedical diagnostics and sensing, neuroscience, and prosthetics. The field of bioelectronics seeks to explore the interfaces between electronics, materials science and other engineering disciplines with biochemistry, biophysics, and general biology.

The collegial environment of this symposium will allow leading experts in the field, rising stars, and novices to discuss a broad array of topics. Additional information can be found at asilomarbioelectronics.org.

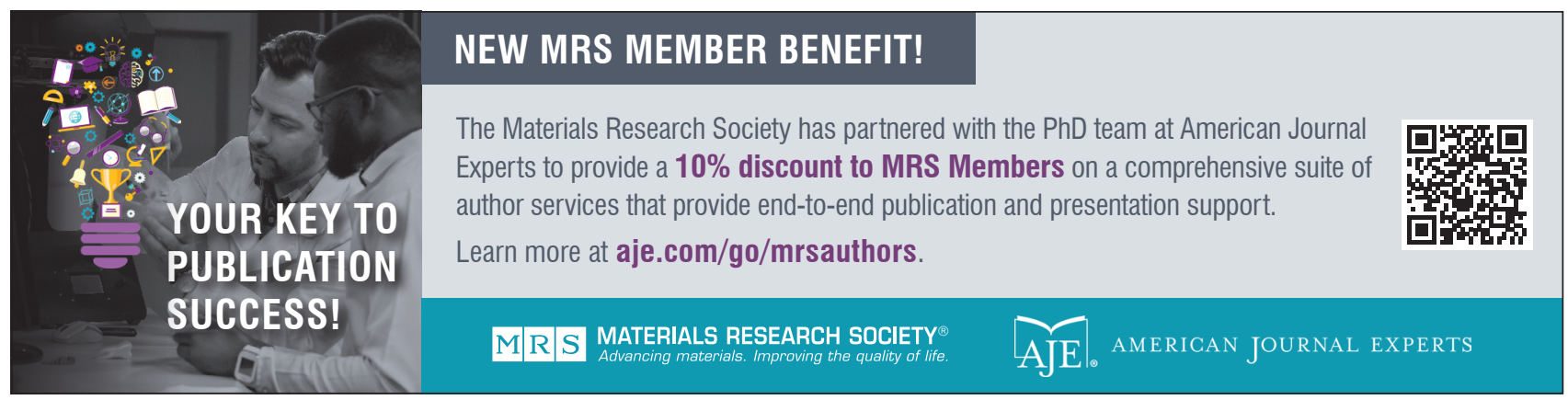

